\title{
Delay-dependent Robust Stability of Uncertain Discrete-Time Switched Systems
}

\author{
Changcheng Xiang (Corresponding author) \\ Department of Computer Science, Aba Teacher College \\ Wenchuan 623000, China \\ E-mail: changcheng96@gmail.com \\ Shouming Zhong \& Lianglin Xiong \\ School of Applied Mathematics, University of Electronic Science and Technology of China \\ Chengdu 610054, China
}

The research is financed by the National Natural Scienec Foundation of China (NSFC-60736029)

\begin{abstract}
This paper address the problems of robust stability for uncertain discrete-time switched systems. The uncertainty is assumed to be of structured linear fractional from which includes the norm-bounded uncertainty as a special case. By introducing a novel difference inequality, new delay-dependent stability criteria are formulated in terms of linear matrix inequalities(LMIs) which are not contained in known literature. Numerical examples are given to demonstrate the effectiveness of the theoretical results.
\end{abstract}

Keywords: Difference inequality, Discrete-time switched system, Delay-dependent stability, Linear matrix inequality

\section{Introduction}

Switched systems are a class of hybrid dynamical systems consisting of a family of continuous- (or discrete-) time subsystems, and a rule that orchestrates the switching between them. It have gained a great deal of attention mainly because various real-world systems,such as chemical processing (S.Engell, 2000), communication networks, traffic control (R.Horowitz, 2000; C. Livadas, 2000; P.Varaiya, 1993), the control of manufacturing systems (D.Pepyne,2000; M.Song, 2000), and automotive engine control and aircraft control (P.Antsaklis, 2000) can be modeled as switched systems. In the last two decades, there has been increasing interest in stability analysis and controller design for switched systems, the reader is referred to the survey paper (Hai lin, 2009), and the references therein. Beside many researches on the continuous switched system (S.Pettersson, 1997)-(Kim. S, 2006), the discrete switched system has also been considered in many paper (Du D., 2006)-(Yuangong Sun, 2007) and see the references therein. It has been recognized that time delays, which are the inherent features of many physical process, are the big sources of instability and poor performances. For time delay systems, stability criteria are usually classified into two types: delay-independent criteria and delay-dependent ones. In general, delay-independent criteria are conservative since they can not handle the systems whose stability depends on the size of time delay. Recently, (Yuangong Sun, 2006) and (Yuangong Sun, 2007) obtained the delay dependent stability condition of uncertain discrete-time switched systems, However, the results of discrete delay is small to some extent. It may be improved significantly with some useful approaches, this has motivated our research.

In this paper, we are interested in establishing delay-dependent stability criteria in terms of linear matrix inequalities (LMIs) for the uncertain discrete-time switched delay systems under arbitrary switching sequences. The main idea of our method is inspired by Zhang's recent work (Xianming Zhang, 2006), where some novel integrate inequalities is introduced for stability analysis and controller synthesis of continuous deterministic delay systems. We extend this approach to uncertain discrete-time switched delay systems based on the constructed switched Lyapunov functionals (J.Daafouz, 2002). The advantage of the introduction of difference inequality lies in that it considerably reduces the conservatism entailed in the previously developed transformation methods since it isn't transform the systems which could introduce additional dynamics in the sense defined in ( Gu. K., 2001). Another important idea of the proposed method is that some free weighting matrices are introduced properly to counteract the influence, bringing by the difference inequality, to the 
delays. Note that these advantages are not obtained at the cost of high computational complexity. Finally, numerical examples are given to illustrate the superiority of present result to those in the literature.

This paper is organized as follows. In Section2, we give the problem formulation and introduce an important lemmas to our later results. Section3 is dedicated to stability analysis of switched systems by mean of a switched quadratic Lyapunov function and our lemma. Two numerical evaluations are given in Section4.

\section{Problem preliminaries}

Nomenclature

$R^{n} \quad$ n-dimensional real space

$R^{n \times n} \quad$ set of all real $\mathrm{n}$ by $\mathrm{n}$ matrices

$x^{T}$ or $A^{T} \quad$ transpose of vector x (or matrix A)

$P>0 \quad$ (respectively, $P<0$ ) matrix $P$ is symmetric positive (respectively, negative) definite

$P \geq 0 \quad$ (respectively, $P \leq 0$ ) matrix $P$ is symmetric positive (respectively, negative) semi-definite

* the elements below the main diagonal of a symmetric block matrix

Consider linear switched system in the domain of discrete time:

$$
\begin{aligned}
& x(k+1)=A(k, r(k)) x(k)+B(k, r(k)) x(k-d), \\
& x(s)=\phi(s), \quad s=-d, \ldots,-1,0 .
\end{aligned}
$$

where $x(k) \in R^{n}$ is the system state, $r(k): Z^{+}=\{0,1,2, \ldots\} \rightarrow \mathscr{N}=\{1,2, \ldots, N\}$ is the control signal. $d$ denote the delay of the system. $\phi:\{-d,-d+1, \ldots, 0\} \rightarrow R^{n}$ represents the initial condition. For each $i \in \mathscr{N}$, the system matrices are assumed to be uncertain and satisfy:

$$
\begin{aligned}
{\left[\begin{array}{ll}
A(k, i) \quad B(k, i)
\end{array}\right] } & =\left[\begin{array}{cc}
A_{i} & B_{i}
\end{array}\right]+H_{i} \triangle\left[\begin{array}{ll}
E_{i 1} & E_{i 2}
\end{array}\right] \\
\triangle & =[I-F(k) J]^{-1} F(k) \\
0 & <I-J J^{T}
\end{aligned}
$$

where $A_{i}, B_{i}$ are constant matrices that describe the $i$ th nominal mode, $H_{i}, E_{i 1}$ and $E_{i 2}$ are given constant matrices which characterize the structure of the uncertainty, and the admissible uncertain matrix $F(k)$ satisfies

$$
F^{T}(k) F(k) \leqslant I
$$

for $k \in Z^{+}$.

The linear fractional parametric uncertainties have been investigated in the robust control setting as related in (Du D., 2006) It is easy to see that when $J=0$, the linear fractional uncertainty reduces to norm bound one. Notice also that condition (4) guarantees that $I-F J$ is invertible.

We are here interested to establish delay-dependent robust stability criteria for systems (1) by introducing a novel difference inequality and using linear matrix inequality technique. Before giving the main theorem of this paper, we firstly provide the following lemmas which plays an important role in our later development.

Lemma 2.1 (S.-S. Zhou, 2003) Suppose that $\Delta$ is given by (2)-(5), with matrices $M=M^{T}, S$ and $N$ of appropriate dimensions. Then the inequality

$$
M+S \triangle N+N^{T} \triangle^{T} S^{T}<0
$$

holds for any $F$ such that $F F^{T} \leqslant I$, if and only if for some $\delta>0$,

$$
\left(\begin{array}{ccc}
\delta M & S & \delta N^{T} \\
S^{T} & -I & J^{T} \\
\delta N & J & -I
\end{array}\right)<0
$$

Lemma 2.2 (Finsler's lemma) For vector $x \in R^{n}$, matrix $P \in R^{n \times n}$ and $H \in R^{m \times n}$, satisfying $\operatorname{rank}(H)=r<n$, the following statements are equivalent,

(i) $\forall x \neq 0$ and $H x=0$, satisfying $x^{T} P x<0$;

(ii) $\exists X \in R^{n \times m}$, satisfying $P+X H+H^{T} X^{T}$.

Lemma 2.3 For any constant symmetric matrix $Q \in R^{n \times n}, Q=Q^{T}>0$, and any appropriate dimensional matrices,

$$
\begin{gathered}
M_{1} \in R^{n \times n}, M_{2} \in R^{n \times n}, Z=\left(\begin{array}{cc}
Z_{11} & Z_{12} \\
* & Z_{22}
\end{array}\right) \in R^{2 n \times 2 n}, Y=\left[\begin{array}{cc}
M_{1} & M_{2}
\end{array}\right] \in R^{n \times 2 n}, \text { if }\left(\begin{array}{cc}
Q & Y \\
* & Z
\end{array}\right)>0, \text { we have } \\
\quad-2 \sum_{l=k-d}^{k-1} x^{T}(l) Q x(l) \leq \xi^{T}(k)\left(\begin{array}{cc}
\Lambda_{11} & \Lambda_{11} \\
* & \Lambda_{22}
\end{array}\right) \xi(k)
\end{gathered}
$$


with $\xi^{T}(k)=\left[\begin{array}{ll}x^{T}(k) & x^{T}(k-d)\end{array}\right]$, where,

$$
\begin{gathered}
\Lambda_{11}=M_{1}+M_{1}^{T}+d Z_{11}+Q+d M_{1}^{T} Q^{-1} M_{1}, \\
\Lambda_{12}=-M_{1}^{T}+M_{2}+d Z_{12}+d M_{1}^{T} Q^{-1} M_{2}, \\
\Lambda_{22}=-M_{2}-M_{2}^{T}+d Z_{22}-Q+d M_{2}^{T} Q^{-1} M_{2} .
\end{gathered}
$$

Proof. With the fact,

$$
x(k)-x(k-d)-\sum_{l=k-d}^{k-1}(x(l+1)-x(l))=0,
$$

$\forall N_{1}, N_{2} \in R^{n \times n}$, we have,

$$
\begin{aligned}
0 & =2\left[x^{T}(k) N_{1}^{T}+x^{T}(k-d) N_{2}^{T}\right]\left[x(k)-x(k-d)-\sum_{l=k-d}^{k-1}(x(l+1)-x(l))\right] \\
& =2 \xi^{T}(k) N^{T}\left[\begin{array}{ll}
I & -I
\end{array}\right] \xi(k)-2 \xi^{T}(k) N^{T} \sum_{l=k-d}^{k-1} x(l+1)+2 \xi^{T}(k) N^{T} \sum_{l=k-d}^{k-1} x(l)
\end{aligned}
$$

where $N=\left[\begin{array}{ll}N_{1} & N_{2}\end{array}\right], \xi^{T}(k)=\left[\begin{array}{ll}x^{T}(k) & x^{T}(k-d)\end{array}\right]$, by using the Moon's inequality (Moon Y.S., 2001), we have,

$$
\begin{aligned}
-2 \xi^{T}(k) N^{T} \sum_{l=k-d}^{k-1} x(l+1) \leqslant & \sum_{l=k-d}^{k-1}\left(\begin{array}{c}
x(l+1) \\
\xi(k)
\end{array}\right)^{T}\left(\begin{array}{cc}
Q & Y-N \\
Y^{T}-N^{T} & Z
\end{array}\right)\left(\begin{array}{c}
x(l+1) \\
\xi(k)
\end{array}\right) \\
= & \sum_{l=k-d}^{k-1} x^{T}(l+1) Q x(l+1)+d \xi^{T}(k) Z \xi^{T}(k) \\
& +2 \xi^{T}(k)\left(Y^{T}-N^{T}\right)\left[\begin{array}{ll}
I & -I
\end{array}\right] \xi(k) \\
& +2 \xi^{T}(k)\left(Y^{T}-N^{T}\right) \sum_{l=k-d}^{k-1} x(l)
\end{aligned}
$$

Substitute (7) into (6), and with the fundamental inequality, we get

$$
\begin{aligned}
0 \leqslant & 2 \xi^{T}(k) Y^{T}\left[\begin{array}{ll}
I & -I
\end{array}\right] \xi(k)+d \xi^{T}(k) Z \xi(k)+2 \xi^{T}(k) Y^{T} \sum_{l=k-d}^{k-1} x(l) \\
& +\sum_{l=k-d}^{k-1} x^{T}(l+1) Q x(l+1) \\
\leqslant & 2 \xi^{T}(k) Y^{T}\left[\begin{array}{ll}
I & -I
\end{array}\right] \xi(k)+d \xi^{T}(k) Z \xi(k)+d \xi^{T}(k) Y^{T} Q^{-1} Y \xi(k) \\
& +\sum_{l=k-d}^{k-1} x^{T}(l) Q x(l)+\sum_{l=k-d}^{k-1} x^{T}(l+1) Q x(l+1) \\
= & 2 \xi^{T}(k) Y^{T}\left[\begin{array}{ll}
I & -I
\end{array}\right] \xi(k)+d \xi^{T}(k) Z \xi(k)+d \xi^{T}(k) Y^{T} Q^{-1} Y \xi(k) \\
& +2 \sum_{l=k-d}^{k-1} x^{T}(l) Q x(l)+\xi^{T}(k)\left(\begin{array}{cc}
Q & 0 \\
0 & -Q
\end{array}\right) \xi(k)
\end{aligned}
$$

it can easy be seen from this that the conclusion is true.

\section{Main results}

In this section, we present asymptotically stability criteria dependent on delays for the uncertain discrete-time switched systems described by (1) and (2) with strict LMI approaches.

For system (1), we define the following switched Lyapunov function :

$$
V(k, x(k))=x^{T}(k) P_{r(k)} x(k)+2 \sum_{\theta=-d+1}^{0} \sum_{l=k-1+\theta}^{k-1} x^{T}(l) Q x(l)
$$

with $P_{1}, P_{2}, \ldots, P_{N}, Q$ being symmetric positive definite matrices. 
If such a Lyapunov function exists and its difference $\Delta V(k, x(k))=V(k+1, x(k+1))-V(k, x(k))$ is negative definite along the solution of (1), the the origin of the system(1) is globally asymptotically stable as shown by the following general lemma.

Lemma 3.1 (M. Vidyasagar, 1993) The equilibrium 0 of

$$
x(k+1)=f(x(k))
$$

is globally uniformly asymptotically stable if there is a function $V: \mathbb{Z}^{+} \times \mathbb{R}^{\ltimes} \rightarrow \mathbb{R}$ such that,

(i) $V$ is a positive definite function, decrescent, and radially unbounded;

(ii) $\Delta V(k, x(k))=V(k+1, x(k+1))-V(k, x(k))$ is negative definite along the solution of (9).

For the asymptotically stability of systems described by (1), we have the following result.

Theorem 3.1 The systems (1) is asymptotically stability, if there exist symmetric matrices $P_{1}, P_{2}, \ldots, P_{N}, Q, Z_{11}, Z_{22} \in$ $R^{n \times n}$ and any appropriate dimensional matrices $G_{i}, T_{i}, U_{i}, M_{1}, M_{2} \in R^{n \times n}$, such that the following LMIs holds,

$$
\begin{gathered}
\left(\begin{array}{ccc}
Q & M_{1} & M_{2} \\
* & Z_{11} & Z_{12} \\
* & * & Z_{22}
\end{array}\right)>0, \\
\Gamma=\left(\begin{array}{cccc}
\Gamma_{11} & \Gamma_{12} & \Gamma_{13} & 0 \\
* & \Gamma_{22} & \Gamma_{23} & d M_{1} \\
* & * & \Gamma_{33} & d M_{2} \\
* & * & * & -d Q
\end{array}\right)<0, \quad i, j \in \mathscr{N},
\end{gathered}
$$

where

$$
\begin{gathered}
\Gamma_{11}=P_{j}-G_{i}^{T}-G_{i}, \quad \Gamma_{12}=G_{i} A(k, i)-U_{i}^{T}, \quad \Gamma_{13}=G_{i} B(k, i)-W_{i}^{T}, \\
\Gamma_{22}=-P_{i}+M_{1}+M_{1}^{T}+U_{i} A(k, i)+A^{T}(k, i) U_{i}^{T}+(2 d+1) Q+d Z_{11}, \\
\Gamma_{23}=-M_{1}^{T}+M_{2}+U_{i} B(k, i)+A^{T}(k, i) W_{i}^{T}+d Z_{12}, \\
\Gamma_{33}=-M_{2}-M_{2}^{T}-Q+W_{i} B(k, i)+B^{T}(k, i) W_{i}^{T}+d Z_{22} .
\end{gathered}
$$

Proof. Choose a switching Lyapunov functional candidate for systems (1) as following:

$$
V(k, x(k))=x^{T}(k) P_{r(k)} x(k)+2 \sum_{\theta=-d+1}^{0} \sum_{l=k-1+\theta}^{k-1} x^{T}(l) Q x(l)
$$

Let the mode at time $k$ and $k+1$ be $i$ and $j$, respectively. That is, $r(k)=i$ and $r(k+1)=j$ for any $i, j \in \mathscr{N}$. Along the solution of (1), and using Lemma 2.3, we have

$$
\begin{aligned}
\Delta V(k, x(k))= & V(k+1, x(k+1))-V(k, x(k)) \\
= & x^{T}(k+1) P_{j} x(k+1)-x^{T}(k) P_{i} x(k)+2 d x^{T}(k) Q x(k) \\
& -2 \sum_{l=k-d}^{k-1} x(l)^{T} Q x(l)+2 d x^{T}(k) Q x(k)-2 \sum_{l=k-d}^{k-1} x(l)^{T} Q x(l) \\
\leqslant & \zeta^{T} \Phi(i, j) \zeta
\end{aligned}
$$

where $\zeta^{T}=\left(\begin{array}{lll}x^{T}(k+1) & x^{T}(k) \quad x^{T}(k-d)\end{array}\right) \neq 0$, and

$$
\Phi(i, j)=\left(\begin{array}{lll}
P_{j} & 0 & 0 \\
* & \Phi_{1} & \Phi_{2} \\
* & * & \Phi_{3}
\end{array}\right)
$$

with

$$
\begin{gathered}
\Phi_{1}=-P_{i}+M_{1}+M_{1}^{T}+(2 d+1) Q+d Z_{11}+d M_{1}^{T} Q^{-1} M_{1}, \\
\Phi_{2}=-M_{1}^{T}+M_{2}+d Z_{12}+d M_{1}^{T} Q^{-1} M_{2}, \quad \Phi_{3}=-Q-M_{2}-M_{2}^{T}+d Z_{22}+d M_{2}^{T} Q^{-1} M_{2},
\end{gathered}
$$

applying Schur's complement (Boyd S., 1993), (11) is equivalent to the following,

$$
\Phi(i, j)+\left(\begin{array}{c}
G_{i} \\
U_{i} \\
W_{i}
\end{array}\right)\left(\begin{array}{ccc}
-I & A_{i} & B_{i}
\end{array}\right)+\left(\begin{array}{c}
-I \\
A_{i}^{T} \\
B_{i}^{T}
\end{array}\right)\left(\begin{array}{ccc}
G_{i}^{T} & U_{i}^{T} & W_{i}^{T}
\end{array}\right)<0
$$


Therefore, we have $\Delta V(k, x(k)) \leqslant \zeta^{T} \Phi(i, j) \zeta<0$ for all $k \geqslant 0$ from the Finsler's lemma. This completes the proof of Theorem1 according to Lemma 3.1.

Remark 3.1 As is well known, it could bring conservativeness inevitably if one use inequality analysis technique to analyze the stability of delay systems. In this paper, it may reduce the conservativeness of our results by introducing some free weighting matrices appropriately with Finsler's lemma.

Remark 3.2 It should be noted that Theorem 3.1 is obtained by using the Lyapunov functional $V(k, x(k))$ given by (8). It is clear that when $P_{r(k)}=P$ for any $i \in\{1,2, \cdots, N\}, V(k, x(k))$ becomes $V(k, x(k))=x^{T}(k) P x(k)+2 \sum_{\theta=-d+1}^{0} \sum_{l=k-1+\theta}^{k-1} x^{T}(l) Q x(l)$, which is called a single quadratic function and has been widely used in research work on this topic. Compared with their results, we can see that our results are more general and less conservative by Example 1.

Considered the uncertainty described by (2)-(5), similar to the proof of Theorem 3.1, we can obtain the following Corollary.

Corollary 3.1 The systems (1) with uncertainty as above mentioned is robust asymptotically stability, if there exist constants $\delta_{i}>0$, symmetric matrices $P_{1}, P_{2}, \ldots, P_{N}, Q, Z_{11}, Z_{22} \in R^{n \times n}$ and any appropriate dimensional matrices $G_{i}, T_{i}, U_{i}, M_{1}, M_{2} \in R^{n \times n}$, such that the following LMIs holds,

$$
\begin{gathered}
\left(\begin{array}{ccc}
Q & M_{1} & M_{2} \\
* & Z_{11} & Z_{12} \\
* & * & Z_{22}
\end{array}\right)>0 \\
\end{gathered}=\left(\begin{array}{cccccc}
\delta_{i} \Psi_{11} & \delta_{i} \Psi_{12} & \delta_{i} \Psi_{13} & 0 & G_{i} H_{i} & 0 \\
* & \delta_{i} \Psi_{22} & \delta_{i} \Psi_{23} & \delta_{i} d M_{1} & U_{i} H_{i} & \delta_{i} E_{i 1}^{T} \\
* & * & \delta_{i} \Psi_{33} & \delta_{i} d M_{2} & W_{i} H_{i} & \delta_{i} E_{i 2}^{T} \\
* & * & * & -\delta_{i} d Q & 0 & 0 \\
* & * & * & * & -I & J^{T} \\
* & * & * & * & * & -I
\end{array}\right)<0
$$

where

$$
\begin{gathered}
\Psi_{11}=P_{j}-G_{i}^{T}-G_{i}, \quad \Psi_{12}=G_{i} A_{i}-U_{i}^{T}, \quad \Psi_{13}=G_{i} B_{i}-W_{i}^{T}, \\
\Psi_{22}=-P_{i}+M_{1}+M_{1}^{T}+U_{i} A_{i}+A_{i}^{T} U_{i}^{T}+(2 d+1) Q+d Z_{11}, \\
\Psi_{23}=-M_{1}^{T}+M_{2}+U_{i} B_{i}+A_{i}^{T} W_{i}^{T}+d Z_{12}, \\
\Psi_{33}=-M_{2}-M_{2}^{T}-Q+W_{i} B_{i}+B_{i}^{T} W_{i}^{T}+d Z_{22} .
\end{gathered}
$$

Proof. Using the uncertain condition (2), we have

$$
\begin{aligned}
\Gamma= & \left(\begin{array}{cccc}
\Gamma_{11} & \Gamma_{12} & \Gamma_{13} & 0 \\
* & \widetilde{\Gamma}_{22} & \widetilde{\Gamma}_{23} & d M_{1} \\
* & * & \widetilde{\Gamma}_{33} & d M_{2} \\
* & * & * & -d Q
\end{array}\right)+\left(\begin{array}{c}
G_{i} H_{i} \\
U_{i} H_{i} \\
W_{i} H_{i} \\
0
\end{array}\right) \Delta\left(\begin{array}{llll}
0 & E_{i 1} & E_{i 2} & 0
\end{array}\right) \\
& +\left(\begin{array}{c}
0 \\
E_{i 1}^{T} \\
E_{i 2}^{T} \\
0
\end{array}\right) \Delta^{T}\left(\begin{array}{llll}
H_{i}^{T} G_{i}^{T} & H_{i}^{T} U_{i}^{T} & H_{i}^{T} W_{i}^{T} & 0
\end{array}\right)<0
\end{aligned}
$$

where $\widetilde{\Gamma}_{22}, \widetilde{\Gamma}_{23}$ and $\widetilde{\Gamma}_{33}$ are taken from $\Gamma_{22}, \Gamma_{23}$ and $\Gamma_{33}$ in theorem 3.1 by replacing $A(k, i)$ and $B(k, i)$ with $A_{i}$ and $B_{i}$ respectively. Using lemma 1 , a sufficient condition guaranteeing $\Gamma<0$ is that there exists positive constants $\delta_{i}$ such that (13) and (14) are hold, which completes this proof.

\section{Numerical examples}

In order to show the effectiveness of the approaches presented in Section 3, in this section, two numerical examples are provided.

Example 1. Consider the uncertain systems described by (1) and (2) $\mathscr{N}=1,2$ and

$$
\begin{aligned}
& A_{1}=\left(\begin{array}{cc}
0.8 & 0.2 \\
0 & 0.91
\end{array}\right), \quad B_{1}=\left(\begin{array}{cc}
0.3 & a \\
b & 0.58
\end{array}\right), \quad E_{11}=E_{12}=0.01 I, H_{1}=c I \\
& A_{2}=\left(\begin{array}{cc}
-0.1 & 0 \\
-0.1 & -0.1
\end{array}\right), \quad B_{2}=\left(\begin{array}{cc}
0.12 & 0 \\
0.11 & 0.11
\end{array}\right), E_{21}=E_{22}=0.01 I, H_{2}=c I
\end{aligned}
$$


It becomes nominal systems if we set $c=0$. In this case, when $a=0, b=0$, using Theorem 1 , both the results in (Yuangong Sun, 2006) and our results are same, viz. $d \leqslant 1$. Whereas, when $a=0.2, b=0.1$, the delay $d$ can be obtained as much as 21 by theorem1, while $d$ in (Yuangong Sun, 2006) remain as $d \leqslant 1$. This comparison shows that our result is much less conservative than that in (Yuangong Sun, 2006).

For comparison, let $J=0$, applying corollary 3.1 to this example shows that the system is robust stable for $d=1$ as $a=b=0, c=0.1$, which is much less than (Yuangong Sun, 2007) whose results is $d \leqslant 5$. However, as $a \neq 0, b \neq 0$ our results is much less conservative than that in (Yuangong Sun, 2007). Take $a=0.2, b=0.1, c=0.1$ for example, we can obtain the system is robust stable for $d \leqslant 11$ while its results in (Yuangong Sun, 2007) is $d \leqslant 5$ which has not changed again. And we can get

$$
\begin{array}{rlr}
G_{1}=\left(\begin{array}{cc}
11.2108 & 5.9572 \\
5.9967 & 39.8991
\end{array}\right), & G_{2}=\left(\begin{array}{cc}
157.8184 & -3.8026 \\
-5.8056 & 0.6278
\end{array}\right), & U_{1}=\left(\begin{array}{cc}
-9.8902 & -10.5418 \\
-7.6314 & -37.0833
\end{array}\right), \\
U_{2}=\left(\begin{array}{cc}
15.1804 & -0.3973 \\
-0.3151 & 0.0343
\end{array}\right), & W_{1}=\left(\begin{array}{cc}
-3.9469 & -5.7376 \\
-5.7395 & -24.3965
\end{array}\right), & W_{2}=\left(\begin{array}{cc}
-18.7054 & 0.3828 \\
0.5412 & -0.0722
\end{array}\right), \\
P_{1}=\left(\begin{array}{cc}
1.0008 & -0.1025 \\
-0.1025 & 0.0172
\end{array}\right), & P_{2}=\left(\begin{array}{cc}
0.0414 & -0.0264 \\
-0.0264 & 0.0169
\end{array}\right), & Q=\left(\begin{array}{cc}
0.0027 & -0.0012 \\
-0.0012 & 0.0007
\end{array}\right) .
\end{array}
$$

This comparison shows that our result is also less conservative than that in (Yuangong Sun, 2007) when $B_{1}$ is not a diagonal matrix. This is also shows that our results and that in (Yuangong Sun, 2007) are not contain each other.

Example 2. Consider the systems described by (1) and assumed to have two modes,i.e., $\mathscr{N}=1,2$ with

$A(k, 1)=\left(\begin{array}{ll}0.62 & 0.27 \\ 0.13 & 0.91\end{array}\right), \quad B(k, 1)=\left(\begin{array}{ll}0.31 & 0.23 \\ 0.12 & 0.58\end{array}\right), \quad A(k, 2)=\left(\begin{array}{cc}-0.25 & 0.36 \\ -0.18 & -0.71\end{array}\right), \quad B(k, 2)=\left(\begin{array}{ll}0.12 & 0.21 \\ 0.15 & 0.11\end{array}\right)$.

Thus, we apply Theorem 3.1 to this example shows that the system is asymptotical stable for $d \leqslant 5$. It shows that our results are effective. On the other hand, by solving the inequalities(10) and (11),we get

$$
\begin{gathered}
G_{1}=1.0 \times 10^{3}\left(\begin{array}{cc}
2.2917 & -0.1749 \\
-1.1044 & 0.5517
\end{array}\right), \quad G_{2}=1.0 \times 10^{3}\left(\begin{array}{cc}
4.2759 & 2.7289 \\
2.1015 & 4.2261
\end{array}\right), \quad U_{1}=\left(\begin{array}{cc}
270.6635 & -831.4655 \\
-396.6739 & 49.6896
\end{array}\right), \\
U_{2}=1.0 \times 10^{3}\left(\begin{array}{cc}
-0.1093 & 2.0249 \\
1.6872 & 2.4878
\end{array}\right), \quad P_{1}=1.0 \times 10^{3}\left(\begin{array}{cc}
3.9212 & -0.8476 \\
-0.8476 & 1.0968
\end{array}\right), \quad Q=\left(\begin{array}{cc}
236.7487 & 10.0861 \\
10.0861 & 13.6549
\end{array}\right), \\
P_{2}=1.0 \times 10^{3}\left(\begin{array}{cc}
2.5082 & -1.2497 \\
-1.2497 & 0.6600
\end{array}\right), \quad W_{1}=\left(\begin{array}{cc}
-34.6963 & -26.6685 \\
-315.5136 & -151.8544
\end{array}\right), \quad W_{2}=\left(\begin{array}{cc}
-420.9922 & -356.7214 \\
-871.1593 & -971.3711
\end{array}\right),
\end{gathered}
$$

This example also shows that our results obtained in this paper are effectiveness.

\section{Conclusion}

The robust stability for uncertain discrete-time switched systems with has been investigated. Based on the switched Lyapunov functional approach, combined with the introduced difference inequality and free matrix method, delay dependent stability criteria have been established in form of LMIs. Numerical examples have shown significant improvements over some existing results.

\section{References}

C. Livadas, J. Lygeros, N. -A. Lynch. (2000). High-level modeling and analysis of the traffic alert and collision avoidance system(TCAS). Proc. IEEE, 88, 926-948.

D. -S. Du, B. Jiang, P. Shi, S.-S. Zhou. (2006). Robust $1_{2}-1_{\infty}$ control for uncertain discrete-time switched systems with delays. Circuits Syst. Signal Process., 25, 729-744.

D. Pepyne, C. Cassandaras. (2000). Optimal control of hybrid systems in manufacturing. Proc. IEEE, 88, $1008-1122$.

H. Lin, G.-S. Zhai, P.-J. ANTSAKLIS. (2003). Robust stability and disturbance attenuation analysis of a class of networked control systems. Proceedings of the 42th IEEE Conference on Decision and Control, Maui, Hawaii USA.

H. Lin, P.-J. ANTSAKLIS. (2009). Stability and Stabilizability of Switched Linear Systems: A Survey of Recent Results. IEEE Transactions on Automatic Control, 54, 308-322.

J. Daafouz, P. Riedinger, C. Lung. (2002). Stability analysis and control synthesis for switched systems: a switched Lyapunov function approach. IEEE Trans. Automat. Control, 47, 1883-1887.

K.-Q. Gu, S.-I. Niculescu. (2001). Further remarks on additional dynamics in various model transformations of linear delay systems. IEEE Trans Automat Contr, 46, 497-500. 
K. Hu, J. Yuan. (2009). Improved robust Ho filtering for uncertain discrete-time switched systems. IET Control Theory Appl., 3, 315-324.

L. -Q. Zhang, Y. Shi, T.-W. Chen, B. Huang. (2005). A new method for stabilization of networked control systems with random delays. IEEE Transactions on Automatic Control, 50, 1177-1181.

L. Hetel, J. Daafouz, C. Iung. (2008). Equivalence between the Lyapunov-Krasovskii functionals approach for discrete delay systems and that of the stability conditions for switched systems. Nonlinear Analysis: Hybrid Systems, 2, 697-705.

M. -S. Branicky. (1998). Multiple lyapunov functions and other analysis tools for switched and hybrid systems. IEEE Trans. Automat. Contr., 43, 475-482.

M. Paolo, S. Mario, D. Jamal. (2007). On stability analysis of linear discrete-time switched systems using quadratic Lyapunov functions. Proceedings of the 46th IEEE Conference on Decision and Control New Orleans, LA, USA, pp.12-14.

M. Song, T. Tran, N. Xi. (2000). Integration of task scheduling, action planning, and control in robotic manufacturing systems. Proc. IEEE, 88, 1097-1107.

M. Vidyasagar. (1993). Nonlinear Systems Analysis, Prentice-Hall International Editions, Englewood Cliffs, NJ.

M. Wicks, P. Peleties, D. DeCarlo. (1998). Switched Controller Synthesis for the Quadratic Stabilization of A Pair of Unstable Linear systems. European Journal of Control, 4, 140-147.

P. Antsaklis. (2000). Special issue on hybrid systems: Theory and applications-A brief introduction to the theory and applications of hybrid systems. Proc. IEEE, 88, 887-897.

P. Varaiya. (1993). Smart cars on smart roads: Problems of control. IEEE Trans. Autom. Contr., 38, $195-207$.

R. Horowitz, P. Varaiya. (2000). Control design of an automated highway system. Proc. IEEE, 88, 913-925.

S.-H. Lee, T.-H. Kim, J.-T. Lim. (2000). A new stability analysis of switched systems. Automatica, 36, 917-922.

S. -S. Zhou, J. Lam. (2003). Robust stabilization of delayed singular systems with linear fractional parametric uncertainties. Circuits Systems Signal Process, 22, 579-588.

S. Boyd, E.-L. Ghaoui, E. Feron, V. Balakrishnan. (1994). Linear matrix inequalities in systems and control theory. Philadelphia: SIAM.

S. Engell, S. Kowalewski, C. Schulz, O. Strusberg. (2000). Continuous discrete interactions in chemical processing plants. Proc. IEEE, 88, 1050-1068.

S. Ibrir. (2008). Stability and robust stabilization of discrete-time switched systems with time-delays: LMI approach. Applied Mathematics and Computation, 206, 570-578.

S. Kim, S.-A. Campbell, X. Liu. (2006). Stability of a Class of Linear Switching Systems with Time Delay. IEEE Transactions on Circuits and Systems I, 53, 384-393.

S. Pettersson, B.Lennartson. (1997). LMI for stability and robustness of hybrid systems,in Proc. Amer. Control Conf., pp. 1714-1718.

Vinícius F. Montagner, Valter J. S. Leite, S. Tarbouriech, Pedro L. D. Peres. (2005). Stability and stabilizability of discrete-time switched linear systems with state delay. American Control Conference, Portland, OR, USA, pp. 38063811.

X.-M. Zhang. (2006). Study on Delay-dependent Robust Control Based on An Integral Inequality Approach, PhD thesis, School of Information Science and Engineering, Central South University.

Y.-G. Sun, L. Wang, G. -M. Xie. (2006). Delay-dependent robust stability and stabilization for discrete-time switched systems with mode-dependent time-varying delays. Applied Mathematics and Computation, 180, 428-435.

Y.-G. Sun, L. Wang, G. -M. Xie. (2007). Delay-dependent robust stability and $H \infty$ control for uncertain discrete-time switched systems with mode-dependent time delays. Applied Mathematics and Computation, 187, 1228-1237.

Y.-S. Moon, P. Park, W.-H. Kwon. (2001). Delay-dependent robust stabilization of uncertain state-delayed systems. International Journal of Control, 74, 1447-1455.

Z.-D. Sun. (2004). Stabilizability and Insensitivity of Switched Linear Systems. IEEE Transactions on Automatic Control, 49, 1133-1137. 